\title{
Valoración económica de los beneficios ambientales de no mercado derivados de la mejora de la calidad del agua: una estimación en aplicación de la Directiva Marco del Agua al Guadalquivir
}

\author{
Julia Martín-Ortega ${ }^{\mathrm{a}}$, Julio Berbel ${ }^{\mathrm{b}}$ y Roy Brouwer ${ }^{\mathrm{c}}$
}

\begin{abstract}
RESUMEN: Esta investigación responde a la necesidad de información económica relativa a los beneficios derivados de la implementación de la Directiva Marco del Agua (DMA). Se plantea una definición práctica del concepto de beneficio ambiental y un escenario de valoración de los beneficios de la mejora de la calidad del agua que no tienen reflejo en el mercado. Esta valoración es necesaria, entre otros aspectos, para la evaluación de la desproporcionalidad de los costes de la DMA. La metodología se aplica a un caso práctico en el Guadalquivir en un ejercicio de valoración contingente y análisis estadístico mediante un modelo Heckman en dos etapas.
\end{abstract}

PALABRAS CLAVE: Beneficios ambientales, costes desproporcionados, Directiva Marco del Agua, modelo Heckman, valoración contingente.

Clasificación JEL: Q25, Q51.

Economic assessment of non-market environmental benefits of water quality: an application of the Water Framework Directive to the Guadalquivir River Basin

SUMMARY: This study contributes to the economic assessment of the benefits derived from the implementation of the Water Framework Directive (WFD). A practical definition of environmental benefits and a valuation scenario of the non-market benefits of water quality improvement is proposed. This economic assessment is needed to asses whether the WFD is disproportionately costly. The contingent valuation method is applied to the Guadalquivir River Basin (Southern Spain), using a two-stage Heckman estimation model.

KEYWORDS: Environmental benefits, disproportionate costs, Water Framework Directive, Heckman model, contingent valuation.

JEL classification: Q25, Q51.

a Basque Centre for Climate Change (BC3).

b Departamento de Economía, Sociología y Política Agrarias. Universidad de Córdoba.

c Institute for Environmental Studies (IVM), VU University of Amsterdam.

Agradecimientos: Esta investigación forma parte del proyecto de la UE: AquaMoney. La encuesta fue financiada por el proyecto CICYT 300083-AGR2006 del Ministerio de Educación y Ciencia. Ha sido posible gracias al Programa de Formación de Doctores de la Junta de Andalucía y la tesis doctoral de la que procede ha sido premiada por el Centro de Estudios Andaluces. Agradecemos al Dr. Manuel Arriaza su asesoramiento en el trabajo de campo.

Dirigir correspondencia a: Julia Martín-Ortega. julia.martin.ortega@bc3research.org

Recibido en septiembre de 2007. Aceptado en octubre de 2008. 


\section{Introducción}

En octubre de 2000 entró en vigor la Directiva 2000/60/CE por la que se establece el marco comunitario de actuación en el ámbito de la política de aguas. El objetivo de la Directiva Marco de Aguas (DMA) es la consecución del buen estado ecológico en las masas de agua de la Unión Europea para el año 2015. Para este objetivo, la Directiva prescribe de forma bastante novedosa la utilización de principios y herramientas económicas, respondiendo a la necesidad de una regulación integrada para alcanzar una gestión sostenible de los recursos hídricos y hacer frente a la creciente presión en la demanda de agua de buena calidad.

El artículo 4 de la DMA, en el que se fijan los objetivos ambientales, permite la derogación de dichos objetivos cuando éstos no puedan conseguirse por motivos de viabilidad técnica o costes desproporcionados. Por costes desproporcionados se entiende en la DMA: que los costes sean desproporcionados respecto a la capacidad financiera para asumirlos, o que los costes del Plan de Cuenca sean desproporcionados respecto a las ventajas (beneficios) que supone la mejora en la calidad ambiental. En ambos casos, corresponde a los Estados Miembros determinar y probar la desproporcionalidad $^{1}$.

En el segundo caso, será necesario comparar el coste de las medidas para el alcance del buen estado ecológico con los beneficios de las mismas, es decir, con los beneficios ambientales derivados del buen estado ecológico.

La introducción de los beneficios ambientales ${ }^{2}$ en la DMA es la primera inclusión de estos conceptos en la legislación ambiental europea. Sin embargo, su definición aún no ha sido suficientemente clarificada ni desde el ámbito institucional ni desde el ámbito científico. Bateman et al. (2006) constatan que hasta el momento se han realizado escasas valoraciones económicas de estos beneficios, en particular en lo que concierne a los valores que no tienen reflejo en el mercado. De hecho, la encuesta realizada por el World Wide Fund for Nature and European Bureau (2006) pone de manifiesto la poca atención que se ha prestado en la primera fase de implementación de la Directiva a los beneficios ambientales, limitándose en la mayor parte de los casos a una mera descripción de los impuestos ambientales existentes ${ }^{3}$.

\footnotetext{
${ }^{1}$ En el primer caso, se trata es de un problema de capacidad financiera que depende del modo en que se repercutan los costes del Plan de Cuenca sobre los usos del agua. Entendemos, como Gómez (2006), que la capacidad financiera insuficiente no es un argumento para rebajar los objetivos ambientales, sino solamente para justificar el aplazamiento de la consecución de los mismos.

2 En la DMA se hace mención a los beneficios ambientales y del recurso. La distinción entre ambos conceptos no está totalmente consensuada ni queda clara en el texto de la Directiva ni en la literatura científica, exhaustivamente revisada por Schaafsma y Brouwer (2006). Es más, para Ferrer y la Roca (2006) la interpretación de los beneficios ambientales y del recurso como categorías separables está en el origen de la confusión conceptual entorno a este asunto. Para una discusión más profunda sobre la distinción entre ambos conceptos se sugiere la lectura de Martín-Ortega (2008).

${ }^{3}$ Para un resumen de los avances europeos en esta línea se puede consultar Strosser (2006).
} 
En este sentido, se considera necesario dar respuesta desde el ámbito científico a las necesidades específicas de información generadas por la implementación de la Directiva Marco del Agua. Esta investigación contribuye a generar información relevante para el proceso de toma de decisiones en la política hídrica bajo el nuevo marco de la DMA, mediante la propuesta de una definición práctica de beneficio ambiental y de un procedimiento de estimación de los beneficios de no mercado derivados de la mejora de la calidad del agua.

El artículo está organizado del siguiente modo: en primer lugar (sección 2) se plantea el objetivo de esta investigación, especificándose las hipótesis de trabajo que se pretenden probar. A continuación (sección 3) se introduce brevemente el caso de estudio (Cuenca del Guadalquivir), la metodología (valoración contingente) y las especificidades del escenario de valoración y de la ejecución de la encuesta. En la cuarta sección se explican las bases del modelo estadístico empleado en el análisis: el modelo en dos etapas Heckman. Finalmente, se presentan los resultados (sección 5) y conclusiones del trabajo (sección 6).

\section{Objetivo e hipótesis de la investigación}

De la implementación de la DMA se esperan de manera generalizada beneficios derivados de la consecución del buen estado ecológico de las aguas [Bateman (2006) y Hanley et al. (2006)]. Parte de estos beneficios tendrán su reflejo en el mercado y podrán ser contabilizados para su inclusión en las distintas operaciones de gestión (por ejemplo: disminución de los costes de extracción si mejora el estado cuantitativo de un acuífero y disminución de los costes de tratamiento del agua para usos domésticos si mejora la calidad de la misma).

Sin embargo, la literatura ha dejado constancia desde hace tiempo de la existencia de valores de no mercado de los recursos naturales en general (Pearce y Turner, 1995) y de los recursos hídricos, en particular. Por ejemplo, Birol et al. (2006) recogen la taxonomía de valores que componen el Valor Económico Total de los recursos hídricos, incluidos los valores de uso indirecto, valores de opción y valores de no uso. Los valores sin reflejo en el mercado han sido tradicionalmente ignorados en la política hídrica, dando lugar a situaciones de asignación ineficiente del recurso [Pearce y Turner (1995), Constanza et al. (1997), Birol et al. (2006), UNESCO (2002), Naciones Unidas (2003)]. La vocación claramente ambiental de la DMA ofrece una oportunidad para incorporar a la gestión hídrica los valores de no mercado para corregir esta situación.

En esta investigación, el beneficio ambiental se define en términos prácticos como el valor (ganancia de bienestar) de los bienes y servicios proporcionados por la Demarcación Hidrográfica como consecuencia del buen estado ecológico. El objetivo de este trabajo es la valoración económica de los beneficios ambientales de no mercado derivados de la implementación de la DMA en la Demarcación Hidrográfica del río Guadalquivir. 
El procedimiento de estimación propuesto se basa en la valoración monetaria de la diferencia de bienestar entre el estado actual y el buen estado ecológico de las aguas, mediante el uso de técnicas de preferencias declaradas.

Se trabaja con las siguientes hipótesis:

- La mejora del estado de las aguas es valorada significativamente por parte de los habitantes de la Cuenca, por tanto, de la consecución del buen estado ecológico se deriva un beneficio para la sociedad (además del beneficio reflejado por el mercado).

- La valoración del público es sensible al alcance de la mejora de la calidad del agua, mayor valor será asignado a mejoras de mayor magnitud [i.e., existe sensibilidad al alcance (Carson, 1997)].

- La mejora de la calidad del agua en el conjunto de la Cuenca generará beneficios distintos para los habitantes de distintas zonas de la misma. Entendemos, como Bateman et al. (2006), que los efectos de la implementación de la DMA no serán geográficamente homogéneos y los beneficios derivados de la mejora de la calidad de las aguas variarán en el espacio no sólo según la distribución y la respuesta física de las masas de agua, sino también en el sentido de la distribución de los beneficiarios.

\section{Valoración de beneficios ambientales de no mercado de la mejora de la calidad del agua en el Guadalquivir}

\subsection{Caso de estudio: la Demarcación Hidrográfica del Guadalquivir}

La Demarcación Hidrográfica del Guadalquivir se localiza al sur de la Península Ibérica (Gráfico 1), comprende una extensión de $57.527 \mathrm{~km}^{2}$ y se distribuye por cuatro Comunidades Autónomas, de las cuales el 90\% corresponde a Andalucía, según datos de la Confederación Hidrográfica del Guadalquivir. Más de 5 millones de habitantes viven en el Guadalquivir.

El territorio de la Cuenca pertenece a tres grandes unidades litológicas: Sierra Morena, Cordillera Bética y Valle del Guadalquivir. El clima es de tipo mediterráneo y viene definido en sus rasgos más elementales por el carácter templado-cálido de sus temperaturas $\left(16,8{ }^{\circ} \mathrm{C}\right.$ como media anual) y por la escasez relativa de sus precipitaciones (media anual de $630 \mathrm{~mm}$ ), así como la irregular distribución espacio-temporal de las mismas.

La Cuenca cuenta con $7.230 \mathrm{Hm}^{3} /$ año de recursos naturales ${ }^{4}$ superficiales (de los cuales el $40 \%$ son recursos disponibles) y $2.576 \mathrm{Hm}^{3} /$ año de recursos naturales subterráneos (de los cuales, el grado global de utilización es del 53\%) según la Confederación Hidrográfica del Guadalquivir (2007). Los principales consumos actuales del agua en la

\footnotetext{
4 "Recurso natural" hace referencia al agua que discurriría a lo largo de todo el año por los cauces si no existieran estructuras de regulación.
} 


\section{GRAFICO 1}

\section{Localización de la Demarcación Hidrográfica del Guadalquivir}

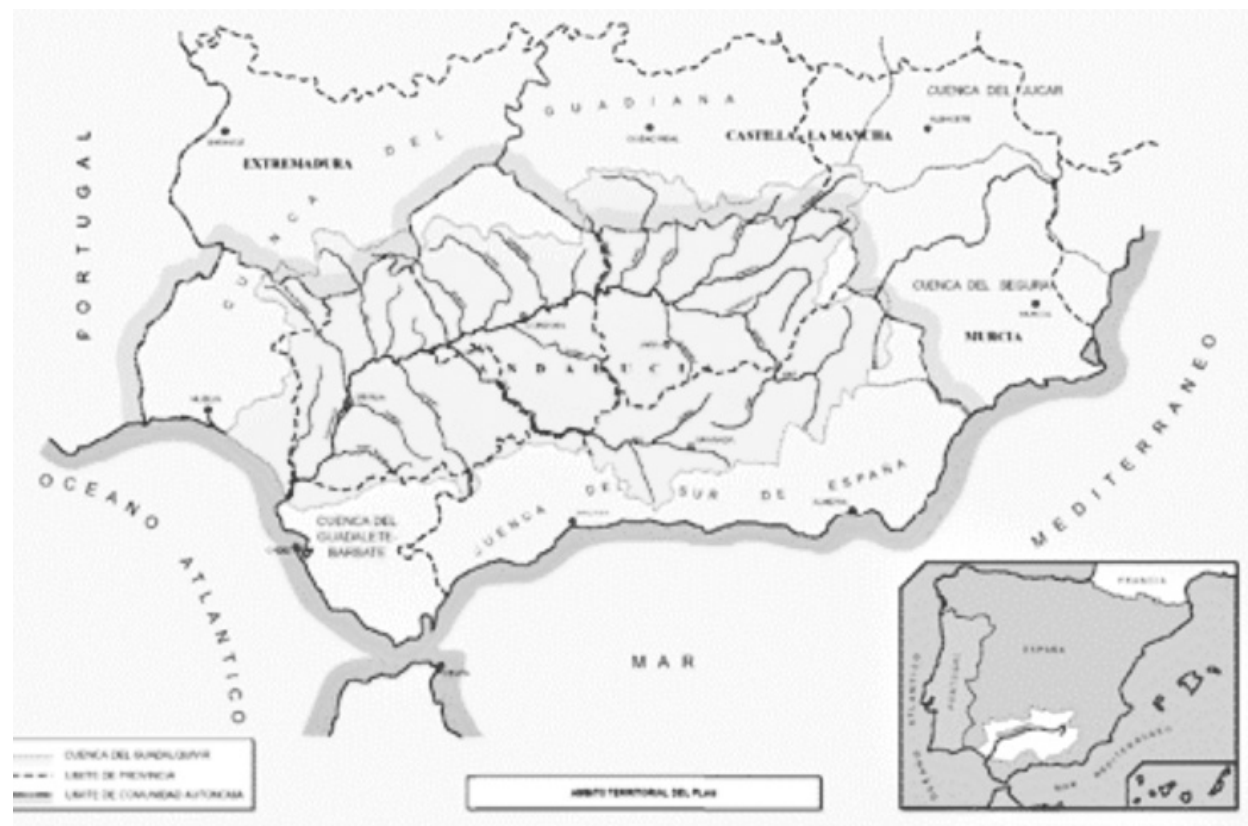

Fuente: Confederación Hidrográfica del Guadalquivir (CHG). www.chguadalquivir.es

Cuenca son la agricultura (87\%), consumo doméstico (9\%), industrial (3\%) y consumo turístico $^{5}(1 \%)$ (Martín-Ortega et al., 2008); y se espera un aumento de la concentración de contaminantes (nitrógeno, fósforo, metales pesados y contaminantes orgánicos) de las aguas de un $30 \%$ en los próximos años (Gutiérrez et al., en prensa).

\subsection{Metodología: la valoración contingente}

Se ha recurrido al método de la valoración contingente, que constituye un caso particular de los procedimientos de valoración basados en la construcción de mercados, en el que se estima el valor de los bienes ambientales a través de la preferencia declarada de los consumidores (Bateman et al., 2002).

\footnotetext{
5 Por uso turístico se entiende, según la caracterización de los usos del agua llevada a cabo también como parte del proceso de implementación de la Directiva Marco del Agua y siguiendo la clasificación propuesta por el Ministerio de Medio Ambiente, Medio Rural y Marino: consumo de agua por uso turismo por viviendas secundarias, turismo industrial (hoteles, pensiones, casas rurales y campings) y campos de golf (Maestu et al. 2008).
} 
El método de valoración contingente se basa en la estimación de la disponibilidad a pagar (o alternativamente la disponibilidad a aceptar) de los individuos por una determinada mejora de la calidad ambiental mediante la asignación hipotética de un determinado precio a dicha mejora. De este modo, la DAP recoge la cantidad de dinero que el individuo tendría que aportar para compensar por el incremento de la utilidad que le proporciona la mejora ambiental.

El uso del método de valoración contingente de los recursos naturales tiene ya un largo recorrido. Carson et al. (1995) repasan más de dos mil estudios de valoración contingente, en su mayoría llevados a cabo en Estados Unidos. En el ámbito europeo, Navrud (1992) revisa estudios de valoración contingente (además de coste de viaje y de precios hedónicos) realizados en numerosos países. Hanley y Wright (1992) pueden ser consultados también para la revisión de los estudios en Reino Unido, que es el país europeo que más experiencia acumula. En el ámbito de los recursos hídricos, se recomiendan los siguientes trabajos de recopilación y revisión: Scotland and Northern Ireland Forum for Environmental Research (2005) para todo tipo de masas de agua en el Reino Unido y los Países Bajos, Brouwer et al. (1999) y Brander et al. (2006) para humedales y Loomis (2000) en Estados Unidos. En cuanto a trabajos que se han realizado en aplicación de las prescripciones de la Directiva Marco de Agua: el trabajo de Brouwer (2004) en el que se estiman los beneficios públicos del buen estado ecológico de las aguas en el río Sheldt mediante valoración contingente y el de Baker et al. (2007) realizado para el Department of Environment, Food and Rural Affairs de Reino Unido.

A pesar de su extendida utilización, tanto la valoración contingente como la propia valoración ambiental en general, permanecen sometidas a debate. Como consecuencia del mismo, los elementos claves de la valoración contingente han sido objeto de continuas revisiones, dando lugar a un marco teórico enriquecido ${ }^{6}$. Los autores concluyen que la mayoría de los problemas argumentados contra la valoración contingente pueden ser resueltos mediante un diseño y aplicación cuidadosos. Por su parte, Shabman y Stephenson (2000) consideran que los defensores y los críticos de la valoración monetaria están reflejando un debate que va más allá del ámbito de los recursos naturales, y que versa sobre el papel que la economía y la cuantificación deben ocupar en la política pública. Estos autores consideran que las críticas a la valoración ambiental cuestionan alguno o ambos supuestos de la valoración ambiental: a) que la elección en mercados (reales o hipotéticos) pueda ser interpretada como reflejo de las preferencias o el valor y $b$ ) que estas interpretaciones deban ser directrices de los procesos de toma de decisión o gestión.

La valoración económica por preferencias declaradas tiene además otra ventaja en el contexto de aplicación de la DMA que se relaciona con la vocación de participación pública de la norma europea. Uno de los principales mecanismos para abordar los conflictos ambientales atendiendo a su complejidad social es la participación pública ${ }^{7}$. En relación explícita con el agua, de la Declaración de

\footnotetext{
${ }^{6}$ Para una profundización en este debate se recomienda la lectura de Carson et al. (2001).

7 Por participación entendemos los foros de intercambio organizados con el propósito de facilitar la comunicación entre el gobierno, los ciudadanos, los agentes implicados, los grupos de interés en relación con una decisión o problema específico (Wittmer et al., 2006).
} 
Dublín $^{8}$ emana el reconocimiento de que la participación pública en todos los niveles de la toma de decisiones relativa a la gestión del agua debe ser tanto un objetivo en sí mismo como un elemento esencial de la gestión (Green, 2003). Este mismo espíritu se encuentra en la redacción de la DMA, cuando en su artículo 14 prescribe la participación pública como un mecanismo dirigido a aumentar la transparencia y la legitimidad de la gestión del agua y ofrecer cauces de diálogo entre usuarios que faciliten la resolución de conflictos (Comisión Europea, 2003).

Se considera que los métodos de preferencias declaradas [que, como Loomis (2000) señala, responden a planteamientos de participación pública] pueden contribuir a que el público sea consultado en relación al proceso de estimación de los beneficios ambientales, en consonancia con la vocación de participación pública de la propia Directiva.

\subsection{Escenario de valoración}

Dos son los elementos básicos del escenario de valoración que se proponen: a) la definición de los niveles de calidad ambiental del agua en relación con su potencialidad de uso y $b$ ) la división de la Cuenca en varias zonas de estudio.

En relación con el primero de los aspectos, la calidad ambiental del agua y sus distintos niveles fueron definidos en términos de los posibles usos del agua (consumo humano y uso natural ${ }^{9}$, uso recreativo y agricultura) y de ciertos niveles de riesgo para la salud de las personas y del entorno (sin riesgo, riesgo bajo, riesgo moderado y riesgo alto), a modo de "escala" de usos y riesgos (calidad muy buena, buena, moderada y mala). Esta definición se basa en la escala de niveles de calidad propuesta por Carson y Mitchell (1993), quienes prepararon para la Agencia de Medio Ambiente estadounidense un estudio de valoración del agua en términos de su susceptibilidad de uso, es decir, la valoración de la calidad de un agua que garantizara las posibilidades de baño (swimminable), pesca (fishable) y navegación $(\text { boatable })^{10}$. Estos usos están, como en el estudio de Carson y Mitchell, así como en estudios posteriores como el de Herberling (2000), organizados de forma jerárquica, de modo que cada nivel superior dentro de la escala incluye los usos incluidos en los niveles inferiores.

\footnotetext{
${ }^{8}$ Conferencia de Naciones Unidas sobre Agua y Medio Ambiente (1992).

9 La denominación "uso natural" es la que se ha encontrado, a través de los grupos de discusión, como más acertada para definir el valor de no uso del agua, quedando definido éste como el uso del agua por el entorno y la vida salvaje.

${ }^{10}$ El trabajo de Carson y Mitchell no está exento de críticas, que podrían hacerse extensibles a esta investigación. Existen trabajos que abogan por que esta simplificación jerárquica no se corresponde siempre con las certidumbres científicas sobre la calidad hídrica e insisten en la necesaria complejidad multidimensional de la valoración del agua [Wesley et al. (2000)]. Sin embargo, la "certidumbre científica" que sirve de base a la crítica de Wesley y sus colegas, parece no haberse alcanzado todavía en el ámbito de la DMA y la definición del estado ecológico de las aguas. De hecho, en el contexto del Ejercicio Europeo de Intercalibración, se reconoce que no existe todavía un acuerdo en la definición de parámetros y umbrales físico-biológicos para la definición de los niveles del estado ecológico.
} 
Se eligió esta escala de calidades-usos tras probar varios enfoques durante la fase de diseño del cuestionario. Los métodos de preferencias declaradas requieren de manera esencial que los individuos encuestados entiendan lo que están valorando. En este sentido, el trabajo de Hanley et al. (2006), que utiliza parámetros físicos de la calidad ecológica de los ríos (vida salvaje saludable y poblaciones vegetales, ausencia de basura en los ríos y buen estado de erosión de los ríos) y que, sin embargo, resultaron no significativos en el análisis, pone de manifiesto la inconveniencia de los mismos para la valoración.

Esta escala de calidad del agua asociada a los usos, además de ser fácilmente comprensible por el público, es coherente con la clasificación que se recoge en la propia $\mathrm{DMA}^{11}$ y permite diseñar escenarios de apoyo a la decisión que sean susceptibles de incluirse fácilmente como insumos de las mismas ${ }^{12}$.

A continuación se recoge la descripción de cada uno de los niveles de calidad del agua, descripciones que fueron acompañadas de pictogramas, que se mostraron permanentemente al encuestado durante todo el ejercicio de valoración (Cuadro 1):

\section{CUADRO 1}

Descripción de los niveles de calidad del escenario de valoración

\begin{tabular}{|c|c|}
\hline $\begin{array}{l}\text { Nivel de } \\
\text { calidad }\end{array}$ & Descripción \\
\hline Muy buena & $\begin{array}{l}\text { Estado natural de las aguas, no hay contaminantes, no hay riesgo para la salud de las per- } \\
\text { sonas ni para el entorno natural; el agua puede usarse para el consumo humano sin nin- } \\
\text { gún tipo de tratamiento; }\end{array}$ \\
\hline Buena & $\begin{array}{l}\text { Puede haber algunos contaminantes, pero a niveles bajos; supone un riesgo bajo tanto } \\
\text { para la salud de las personas como para el entorno natural; se podría usar directamente en } \\
\text { agricultura y uso recreativo (para bañarse y pescar) y sólo sería potable con un trata- } \\
\text { miento previo; }\end{array}$ \\
\hline Moderada & $\begin{array}{l}\text { Agua con contaminantes, pero a unos niveles que permiten usarla para el riego en agri- } \\
\text { cultura; no se puede beber y debe evitarse el baño o comer el pescado procedente de esta } \\
\text { agua; supone un riesgo moderado tanto para la salud de las personas como para el en- } \\
\text { torno y la vida salvaje que depende del entorno; }\end{array}$ \\
\hline Mala & $\begin{array}{l}\text { Agua contaminada, no puede ser usada sin tratamiento; debe evitarse el contacto humano } \\
\text { directo (beber y baño) con ella; supone un riesgo alto tanto para la salud de las personas } \\
\text { como para el entorno }\end{array}$ \\
\hline
\end{tabular}

Fuente: Elaboración propia.

11 Anexo V de la DMA, sección 1.4.2 relativa a la preparación de la monitorización de resultados y clasificación del estado/potencial ecológico.

12 Conviene señalar que hay un elemento clave que no aparece reflejado en el análisis tal y cómo se ha planteado. El buen estado ecológico del agua no se relaciona exclusivamente con sus condiciones de calidad. Es evidente que el mantenimiento de ciertos volúmenes de agua determina de manera fundamental la buena salud de los ecosistemas. Este aspecto cuantitativo es particularmente relevante en nuestra región donde las tendencias apuntan a situaciones futuras insostenibles en cuanto al consumo del agua (MartínOrtega et al., 2008) y muestran escenarios de creciente competencia entre los usos del agua. Siendo conscientes de la importancia de este aspecto cuantitativo, se ha tratado, bajo distintas fórmulas, de incluirlo en esta investigación. Los grupos de discusión y los test pilotos, demostraron, sin embargo, la dificultad de aproximación intelectual al problema por parte de los encuestados cuando se incluían ambos aspectos en un mismo ejercicio de valoración. Este es sin duda un aspecto en el que deberá trabajarse en el futuro. 
En lo que se refiere a la división de la Cuenca en zonas de estudio, como se ha mencionado, una de las hipótesis de trabajo de esta investigación se relaciona con la distinta distribución de los beneficios ambientales en los territorios de la Cuenca. El objetivo impuesto por la DMA es la mejora del estado de las aguas a nivel de Cuenca, pero la situación de partida (status quo) es diferente en distintas zonas de la misma, de modo que consideramos que es esperable que residentes de distintas zonas obtengan beneficios distintos derivados de una mejora global de las condiciones de la demarcación. Es por ello que la Cuenca se dividió en tres zonas de estudio: zona Alto (correspondiente a Sierra Morena y las partes altas de la Cuenca), zona Valle (que incluye los valles del Guadalquivir y el río Genil) y la zona Campiña. A cada una de estas zonas se asignó un determinado nivel de calidad del agua de acuerdo con la escala de calidades-usos anteriormente explicada (ver Cuadro 2). Esta asignación de niveles de calidad ha sido realizada tomando como base la información ofrecida por los informes sobre los artículos 5 y 6 elaborados como parte de la implementación de la DMA (Confederación Hidrográfica del Guadalquivir, 2005) y fue validada por expertos de la Confederación Hidrográfica del Guadalquivir ${ }^{13}$.

\section{CUADRO 2}

Nivel de calidad del agua por zona (status quo)

\begin{tabular}{cc}
\hline Zona & Nivel de calidad agua (status quo) \\
\hline Alto & Buena \\
Valle & Moderada \\
Campiña & Mala \\
\hline
\end{tabular}

Fuente: Elaboración propia.

\subsection{Cuestionario y proceso de muestreo}

El cuestionario diseñado para esta investigación fue el resultado de: $a$ ) una exploración preliminar (consulta a un grupo de 15 expertos del sector y dos grupos de discusión de 6 y 10 personas, respectivamente) y b) tres encuestas piloto (de 40, 40 y 60 entrevistas respectivamente) llevados a cabo durante los meses de mayo a septiembre de 2006. La encuesta definitiva se implementó en el mes de octubre de 2006, por una empresa regional especializada en la aplicación de encuestas de valoración por preferencias declaradas.

El cuestionario se compone de tres bloques principales. El primero de ellos está orientado a la identificación de la relación del individuo con el agua y con los problemas ambientales en general. El segundo bloque constituye el núcleo del ejercicio de valoración. Los encuestados fueron confrontados consecutivamente a dos cuestio-

\footnotetext{
${ }^{13}$ Los mapas fueron evaluados por cuatro expertos de calidad de agua de la Confederación Hidrográfica del Guadalquivir mediante un breve cuestionario.
} 
nes sobre su disponibilidad a pagar por pasar de la situación actual a una situación de una calidad buena primero y muy buena después en el conjunto de la Cuenca ${ }^{14}$. Se recuerda que la escala de calidad se basa en la propuesta por Carson y Mitchell (1993) y se definió en los términos que ya se han especificado en el apartado 3.3. de este artículo (Cuadro 1).

El vehículo de pago elegido para esta valoración fue el incremento anual de la factura del agua ${ }^{15}$. El vehículo de pago es un elemento crucial en las encuestas de valoración contingente, ya que provee el contexto en el que se produce el pago y afecta al modo en que el encuestado responde a la pregunta de elección, pudiendo dar lugar a la aparición de sesgos cuando el vehículo de pago es incorrectamente interpretado o interpretado de manera diferente al investigador (Mitchell y Carson, 1989) ${ }^{16}$.

Otro aspecto fundamental del diseño es el formato de elección. Se ha producido en el ámbito de la economía ambiental un debate sobre el formato de la elección más apropiado [ver por ejemplo Hanley et al. (2003), y Foster y Mourato (2003)], ya que la literatura ha demostrado que distintos formatos de elección dan lugar a diferentes estimaciones de DAP (Carson et al., 2001). Este debate se ha centrado principalmente en la elección entre dos vehículos: el formato abierto (más antiguo) y la elección dicotómica que ofrece estimaciones mayores y menor número de ceros. El formato abierto permite trabajar con muestras de menor tamaño, pero se considera más susceptible de generar respuestas protestas (en general, puede resultar más difícil elegir una cifra que responder sí o no a preguntas concretas) y más dependiente de la credibilidad del escenario. Sin embargo, los estudios de Ready et al. (2001) ponen de manifiesto que el formato abierto muestra las cantidades que los encuestados están dispuestos a pagar con mayor grado de certeza. El panel de expertos que elaboraron el informe para la NOAA sobre la aplicabilidad de la valoración contingente (Arrow et al., 1993) recomendó el formato de elección dicotómica ${ }^{17}$. Desde enton-

${ }^{14}$ Concretamente la pregunta se formuló del siguiente modo: 1) ¿Estaría usted, en principio, dispuesto a pagar una cantidad adicional de dinero para mejorar la calidad del agua en la Cuenca del Guadalquivir?, 2) ¿Puede señalar en la siguiente tarjeta cuánto estaría dispuesto a incrementar al año su factura del agua como máximo durante los próximos diez años para asegurar una calidad buena del agua en toda la Cuenca del Guadalquivir?. Estas preguntas se acompañaron de soporte visual en el que se mostraba el mapa de la Cuenca con el código de color correspondiente (azul para muy buena, verde para buena, amarillo para moderada y rojo para mala). La escala de calidad del agua se mantuvo visible para el encuestado durante toda la entrevista.

15 Durante el proceso de prueba de la encuesta se ensayaron tres vehículos de pago: impuesto de calidad del agua, incremento bimensual de la factura del agua e incremento anual de la factura del agua. Este último fue el que menos respuestas protesta (específicas relativas al vehículo de pago) generó durante las encuestas piloto (3\% frente a 8 y $4 \%$ del y impuesto y la factura bimensual respectivamente).

${ }^{16}$ Según Morrison et al. (2000) una causa común del sesgo por vehículo de pago es que este sea considerado no plausible o cuestionable por el entrevistado. Si tal es el caso, éste puede tratar la encuesta como hipotética y modificar su oferta (DAP) o bien protestar. Para minimizar los posibles sesgos por vehículo de pago Morrison et al. proponen, entre otras técnicas, la recodificación de las respuestas protesta, tal y como se ha realizado en esta investigación.

17 Arrow et al. (1993) también hablan de la necesidad de una muestra de unos 1.000 individuos como apropiada para la elección dicotómica, lo cual puede resultar demasiado costoso en la mayoría de las aplicaciones de valoración. 
ces, la literatura se ha inclinado por el uso de este formato (Carson et al., 2001). Si bien, no hay que olvidar que la elección dicotómica también es susceptible de sesgos tal y como señalan Bateman et al. (1995), derivados de aspectos psicológicos de la elección: cuando el encuestado se enfrenta con bienes con los que no tiene familiaridad, puede interpretar la oferta de la elección dicotómica como una indicación del valor verdadero del bien y producirse un sesgo de anclaje. Además, Balistreri et al. (2001) encuentran que el sesgo hipotético es menor en los formatos abiertos que cerrados. En esta investigación se ha optado por la tarjeta de pago, que ofrece al encuestado una gama de posibles DAP crecientes entre las que elegir, del modo recomendado por Rowe et al. (1996). La tarjeta de pago incluyó cantidades desde 0 a > 300 euros (cantidades seleccionadas de modo que la cantidad máxima fuese mayor que la cantidad máxima de las DAP anticipadas durante las encuestas piloto). La tarjeta de pago combina las ventajas de los formatos abiertos y cerrados a la vez que minimiza el sesgo del punto de partida (Kallas et al., 2007).

Finalmente, el cuestionario incluye un tercer bloque de preguntas destinado a recoger la información socio-demográfica potencialmente relevante para explicar la heterogeneidad de las preferencias sociales. En el apartado de resultados se detallan estas variables.

Se llevó a cabo un muestreo aleatorio por cuotas de edad, sexo y zona (Alto, Valle, Campiña), obteniéndose una muestra representativa (ver el Cuadro 3 que compara las principales características sociodemográficas entre la muestra y la población) formada por 619 residentes de la Demarcación Hidrográfica del Guadalquivir ${ }^{18}$.

Encontramos que la información contenida en la tabla confirma que la muestra es razonablemente representativa del conjunto de la población universo. Si bien es cierto que la población entrevistada presenta un ingreso medio significativamente mayor (I.C. $95 \%=1.840-1.971 € /$ mes). También se observa una ligera sobre-representación significativa de la población con formación universitaria (I.C. 95\% = 16,8$23,2)$.

\section{Análisis estadístico en dos etapas: el modelo Heckman}

Cuando se manejan distribuciones de datos desviadas hacia el valor cero, como ocurre en general con los valores de DAP, las regresiones lineales estándares (OLS)

${ }^{18}$ La población universo considerada es la Demarcación Hidrográfica, ya que es éste el ámbito de aplicación de la DMA. Si bien conviene señalar que los datos utilizados para cotejar la representatividad de la muestra corresponden a Andalucía, ya que no existen o son muy escasos los datos socio-demográficos desagregados a nivel de Demarcación (Martín-Ortega et al., 2008). Se podría argumentar la existencia de beneficios ambientales derivados de la mejora de la calidad del agua también fuera de la Demarcación del Guadalquivir y de la Comunidad Autónoma Andaluza. Por motivos de presupuesto no se pudieron llevar a cabo entrevistas en otros territorios. Sería interesante analizar estos aspectos en investigaciones futuras, si bien, es bastante previsible que en la aplicación práctica de la DMA la estimación de los beneficios ambientales se circunscriba a los ámbitos de las demarcaciones hidrográficas, ya que éstas son las unidades de gestión establecidas por la propia Directiva. 
CUADRO 3

Descripción sociodemográfica: la muestra frente al conjunto de la población

\begin{tabular}{lcc}
\hline \multicolumn{1}{c}{ Variables } & Muestra & Población Andalucía \\
\hline Grupos de edad (\%) & & 32,5 \\
18 -34 años & 34,1 & 48,8 \\
35-59 años & 44,6 & 18,7 \\
$\quad$ 60 años o más & 21,3 & 51,4 \\
Género (\% mujeres de más de 18 años sobre total población & 54,70 & 2,8 \\
de más de 18 años). & 3,3 & 63,8 \\
Tamaño del hogar (media número de personas por hogar) & 66,2 & 1.619 \\
Hogares con niños (\% total hogares) & 1.940 & 17,1 \\
Ingresos medios (€ netos por hogar y mes) & & 69,9 \\
Nivel educativo (\% sobre población total) & 15,3 & 13,0 \\
Sin estudios & 64 & 20 \\
Educación primaria y secundaria & & \\
Educación superior (universitaria) & & \\
\hline
\end{tabular}

Fuente: Elaboración propia.

ofrecen estimaciones inconsistentes de la DAP (Maddala, 1983). En este caso, los modelos Tobit o modelos truncados o censurados (Tobin, 1958; Goldberger, 1964), en los que el rango de la variable dependiente se constriñe entorno a un valor determinado (en este caso hacia el cero), se han propuesto como una mejor alternativa [(Amemiya, 1984), (Halstead et al., 1991), (Seung-Hoon y. et al., 2000)]. Sin embargo, en el modelo Tobit no se tiene en cuenta el posible efecto de auto-selección de la muestra determinado por la voluntad de participar (o no) en el mercado contingente.

Este asunto de la auto-selección lleva a la discusión sobre el tratamiento de las respuestas protesta en el análisis. Cuando el individuo encuestado no se muestra dispuesto a pagar, tal negativa puede corresponder con lo que se conoce como cero genuino o con una respuesta protesta. Los ceros legítimos o genuinos implican que el individuo no está dispuesto a pagar por el bien que se ofrece, y por tanto, y teniendo en cuenta la base de las técnicas de preferencias declaradas, se supone que no valoran el bien (le asignan un valor cero). Las respuestas protestas corresponden a individuos que consideran que el bien tiene un valor superior a cero pero se niegan a revelarlo (Riera, 1994), se niegan a "entrar en el juego" de la valoración.

El procedimiento estándar es el de incluir en el análisis los ceros genuinos y eliminar las respuestas protesta (consúltese Whitehead et al. (1993), Mitchell y Carson (1989) y Jorgensen et al. (1999) a cerca de esta discusión). Sin embargo, como apuntan Shyamsundar y Kramer (1996), Jorgensen y Syme (2000) y 
Sigelman y Zeng (1999), este procedimiento puede afectar a la validez de las estimaciones. Eliminar las respuestas protesta sólo puede considerarse legítimo si el grupo de "protestantes" no es significativamente distinto (en cuanto a las características que influyen en la valoración del bien) del conjunto de la muestra. Si este no es el caso, se produce un sesgo de selección en la muestra que afectará a las estimaciones de DAP. En tal caso, es necesario un modelo que tenga en cuenta este proceso de auto-selección dentro de la propia muestra. Tal es el conocido como modelo de Heckman. Heckman (1979) desarrolló un procedimiento estadístico para tratar la auto-censura de los encuestados. Este modelo se caracteriza por desarrollarse en dos etapas: en la primera se estima una función que especifica la probabilidad de que el encuestado acepte participar en el mercado contingente y, en una segunda etapa, se estima una función específica para la DAP. El modelo de Heckman prueba si la participación en el mercado contingente es aleatoria o no. Si no es aleatoria, los factores que determinan la participación pueden estar correlacionados con los factores que determinan los valores de DAP dando lugar a sobre o subestimaciones de la misma. [1]:

Formalmente, el procedimiento Heckman puede representarse del siguiente modo

$$
E\left(D A P_{i j}\right)=E\left(D A P_{i 2} / Y_{i}, D A P_{i 1}=1\right)=Y_{j} B_{i j}+\varepsilon_{i j}
$$

Donde la variable dependiente consiste en dos indicadores: $D A P_{i 1}$ y $D A P_{i 2}$. $D A P_{i 1}$ es una variable binaria que define la participación (1) o no (0) en el proceso y $D A P_{i 2}$ es la variable continua "cantidad de dinero que se está dispuesto a pagar". $D A P_{i 2}$ sólo es observable si $D A P_{i 1}$ es igual a $1 . \beta_{i j}$ son los coeficientes estimados para cada una de las variables independientes $\left(Y_{i}\right)$, y $\varepsilon_{i j}$ son los términos de error. El sesgo de selección es un problema cuando $\varepsilon_{i 1}$ y $\varepsilon_{i 2}$ están correlacionados (violación de la asunción de que los errores están idéntica e independientemente distribuidos, iid). El procedimiento Heckman controla dicha correlación mediante la inclusión de la razón inversa de Mills durante la primera fase en un modelo Probit y representando la probabilidad de que el encuestado esté en la sub-muestra $D A P_{i 1}=1$ en la regresión que se lleva a cabo en la segunda etapa.

\section{Resultados}

\subsection{Relación del individuo con el agua y los problemas ambientales}

Como resultado de la encuesta, encontramos que el $60 \%$ de los entrevistados se considera interesado o muy interesado en los problemas ambientales, pero tan sólo el $1,1 \%$ es miembro de una organización ecologista o de protección del medio ambiente. 
La gran mayoría de la muestra (más del 90\%) considera que el agua es un problema (muy) importante en España, el $40 \%$ cree que la calidad del agua en el Guadalquivir es mala o deficiente y el $93 \%$ considera que alcanzar el buen estado de las aguas es bastante o muy importante. Por tanto, la población declara un interés por el bien a valorar. La cuestión está en ver si el público está dispuesto a pagar por ello y cuánto.

La relación recreativa de los entrevistados con el agua es escasa: apenas un 10\% de la muestra practica algún tipo de deporte acuático (incluido el baño y la pesca recreativa) en el río.

También se preguntó a los encuestados sobre su factura del agua, con el fin de comparar estos datos con su disponibilidad a pagar. Alrededor del $40 \%$ de la muestra recuerda su factura del agua. Los entrevistados recuerdan haber pagado una media de 264 euros por hogar al año. Esta cifra es bastante próxima a cifras oficiales: el Ministerio de Medio Ambiente, Medio Rural y Marino (2007) ofrece el dato de 308 euros por hogar al año y la Asociación Española de Abastecimiento y Saneamiento de Agua sitúa la factura del agua en 247,5 euros por hogar al año (AEAS, 2003).

Los encuestados fueron primero preguntados por su disponibilidad a pagar en principio para mejorar la calidad del agua sin ninguna especificación adicional. El $39,3 \%$ de la muestra declaró no estarlo. Del total de respuestas negativas, el $84 \%$ fueron catalogadas como ceros legítimos y el 16\% (6,2\% del total de la muestra), se consideraron respuestas protesta, tras el análisis individualizado de cada una de las razones esgrimidas por los encuestados por su negativa a pagar, según la clasificación que se presenta en el Cuadro 4.

El Cuadro 5 muestra los valores medios y principales estadísticas de la respuesta sobre la DAP por la mejora a una calida buena y una calidad muy buena en el conjunto de la Cuenca, después de haber eliminado una respuesta por haberse considerado outlier $^{19}$. Observamos como la disponibilidad media a pagar por un agua de buena calidad en el conjunto de la cuenca es de 31,79 euros al año por hogar, y por un agua de calidad muy buena es de 39,42 euros. Como ocurre con los datos de DAP en general y como se prueba por el test de normalidad que se muestra en el mismo Cuadro 5, ambas cantidad no siguen una distribución normal, poniendo de manifiesto el ya mencionado "desvío" de los datos hacia el valor cero y la imposibilidad de llevar a cabo regresiones lineales estándares.

Se observa que existe una diferencia de aproximadamente 7,2 euros entre ambas disponibilidades a pagar. Es decir, en principio parece que la población está dispuesta a pagar un $24 \%$ más para asegurar que el incremento de la calidad sea hasta una situación muy buena, con respecto a lo que está dispuesta a pagar por una calidad buena como media. Sin embargo, el test Man-Whitney mostró que los dos valores (DAP media buena calidad y DAP media muy buena calidad) no son significativamente distintos ( $\mathrm{p}$-value $=0,152$ ), es decir, no hay diferencia estadística.

19 Para la identificación del outlier se utilizó la función Stem-and-Leaf Plot ofrecida por el software SPSS. La eliminaciónd el outlier no generó el cambio de signo de los coeficientes. 


\section{CUADRO 4}

Respuestas protesta y ceros legítimos

\begin{tabular}{|c|c|c|c|c|c|c|c|}
\hline Ceros legítimos & Obs. & $\begin{array}{l}\% \text { sobre } \\
\text { la } \\
\text { muestra }\end{array}$ & $\begin{array}{c}\% \text { sobre } \\
\text { el total } \\
\text { de ceros } \\
\text { legítimos }\end{array}$ & Protesta $^{20}$ & Obs. & $\begin{array}{l}\text { \% sobre } \\
\text { la } \\
\text { muestra }\end{array}$ & $\begin{array}{c}\% \text { sobre } \\
\text { el total } \\
\text { de } \\
\text { protestas }\end{array}$ \\
\hline La situación es buena & 12 & 1,9 & 5,9 & $\begin{array}{l}\text { Es un problema de la } \\
\text { Administración }\end{array}$ & 18 & 2,9 & 45,0 \\
\hline No puedo pagarlo & 96 & 15,5 & 47,3 & Que pague el contaminador & 4 & 0,6 & 10,0 \\
\hline No creo que sea importante & 2 & 0,3 & 1,0 & $\begin{array}{l}\text { Es un recurso natural, } \\
\text { debería ser gratis }\end{array}$ & 1 & 0,2 & 2,5 \\
\hline $\begin{array}{l}\text { Prefiero gastar el dinero en } \\
\text { otras cosas }\end{array}$ & 19 & 3,1 & 9,4 & No confío en el sistema & 17 & 2,7 & 42,5 \\
\hline Ya pago suficiente & 60 & 9,7 & 29,6 & Total & 40 & 6,5 & 100,0 \\
\hline NSNC & 14 & 2,3 & 6,9 & & & & \\
\hline Total & 203 & 32,8 & 100,0 & & & & \\
\hline
\end{tabular}

Fuente: Elaboración propia.

\section{CUADRO 5}

Disponibilidad a pagar por agua de buena calidad y muy buena calidad: principales estadísticas y test de distribución normal

\begin{tabular}{lcc}
\hline & $\begin{array}{c}\text { DAP por agua de buena calidad } \\
\text { en el conjunto del la Cuenca } \\
(€ \text { por hogar al año) }\end{array}$ & $\begin{array}{c}\text { DAP por agua de muy buena calidad } \\
\text { en el conjunto del la Cuenca } \\
\text { (€ por hogar al año) }\end{array}$ \\
\hline Observaciones válidas & 554 & 550 \\
Media & 31,79 & 39,42 \\
Error típico de la media & 1,90 & 2,37 \\
Mediana & 12 & 20 \\
Mínimo & 0 & 0 \\
Máximo & 300 & 300 \\
Kolmogorov-Smirnov Z* & 5,62 & 5,61 \\
Asymp. Sig. (2-tailed) & 0,000 & 0,000 \\
\hline
\end{tabular}

Fuente: Elaboración propia.

${ }^{20}$ La respuesta "es un problema de la Administración", fue considerada como una respuesta protesta contra el vehículo de pago: la mayoría de las empresas de aguas son empresas públicas, que dependen de la Administración. Si el encuestado considera que la Administración debería ocuparse del asunto, pero no quiere pagar por ello, deducimos que no está interpretando correctamente el vehículo de pago o lo está cuestionando, dando lugar a una respuesta protesta del tipo descrito por Morrison et al. (2000). Las respuestas "que pague el contaminador" y "el agua es un recurso natural, debería ser gratis", comprenden lo que Hanley (1996) llama protesta ética: el encuestado rechaza la valoración por motivos éticos. 
A continuación se presentan los resultados del análisis multivariante realizado en dos etapas mediante el modelo de Heckman ${ }^{21}$. Este análisis permite estudiar la heterogeneidad de la demanda en cuanto a las características socio-demográficas y de comportamiento/percepción de la población. Se incluyen además la variable de identificación del nivel de calidad del agua para la profundización sobre la sensibilidad al alcance de la mejora (hipótesis segunda) y las variables de zona de residencia para probar la hipótesis tercera.

Se observa cómo existen diferencias entre las variables que influyen en la decisión a participar en el mercado contingente (modelo de auto-selección muestral, Cuadro 6) y las variables que influyen en la cantidad que se está dispuesto a pagar (lo que hemos llamado modelo principal, presentado en el Cuadro 7). Analizamos en primer lugar las variables comunes.

Por una parte, y de acuerdo con las expectativas de la teoría económica, mayores ingresos determinan tanto mayor probabilidad de disponibilidad a pagar como mayores cantidades de dinero que se está dispuesto a pagar (en ambos modelos el nivel de ingresos es significativo al $5 \%$ en el primero y al $1 \%$ en el segundo).

El hecho de tener hijos también es un factor determinante en este mismo sentido; las personas con hijos se muestran más disponibles a pagar y están dispuestas a pagar más dinero por la mejora de la calidad del agua en la Cuenca, según los datos recogidos en nuestra encuesta. Esto podría ser interpretado como derivado de un mayor grado de conciencia sobre el disfrute de los bienes ambientales por las generaciones futuras, el llamado valor de legado (Krutilla, 1967; Kosz, 1996).

Tras presentarles el mapa de la situación actual de la calidad del agua (status quo) en el Guadalquivir, los encuestados fueron consultados sobre cuán concientes se consideraban con respecto dicha situación actual. El 34,4\% de la muestra se manifestó más o menos consciente, consciente o muy consciente de la situación actual. Se observa, además, en el Cuadro 6 cómo esta variable influye significativamente (al $5 \%$ ) en la DAP; cuanto mayor es el grado de conciencia sobre la situación actual de la calidad del agua en la Cuenca, mayor es la disponibilidad del individuo a participar en el mercado contingente y mayor es también la cantidad de dinero que está dispuesto a pagar por mejorarla (Cuadro 7).

Existen, sin embargo, ciertos aspectos diferenciales entre ambos modelos. En cuanto al modelo de auto-selección de la muestra (primera fase del modelo de Heckman: disponibilidad a participar en el mercado contingente), se observa como la credibilidad concedida por el individuo al escenario de partida o status quo juega un rol determinante en su participación en el ejercicio de valoración. Cuanto mayor es la credibilidad que el encuestado da al status quo, mayor es su disponibilidad a participar. Esta variable no es significativa en el modelo de DAP (segunda fase del modelo de Heckman). Tal resultado parece lógico; que el individuo crea o no lo que se le plantea influirá en su voluntad de participar en el proceso, pero una vez que ya participa en el mismo la variable credibilidad deja de influir en la cantidad que está dispuesto a pagar.

\footnotetext{
${ }^{21}$ El modelo Heckman se realizó utilizando el software STATA, Versión 10SE.
} 
También es interesante constatar que el hecho de considerar que existen problemas relativos a la cuestión hídrica en la región (variable "el agua es un problema") y el grado de importancia que se da a la mejora de la calidad del agua en la Cuenca (variable "importancia de la calidad del agua") determinan la participación del individuo (aunque no la cantidad de dinero que está dispuesto a pagar).

Finalmente, observamos cómo a mayor nivel educativo del individuo, mayor disponibilidad a pagar en principio (participar) por mejorar la calidad del agua, lo que posiblemente venga determinado por un mayor nivel de conciencia sobre los problemas ambientales y la responsabilidad individual en su solución.

En cuanto al modelo que hemos llamado "principal" (segunda etapa del modelo de Heckman, Cuadro 7), encontramos los siguientes aspectos relevantes:

- La variable que identifica el nivel de calidad bueno y muy bueno ha resultado no significativa (valor $p=0,762$ ), lo que impide confirmar la hipótesis segunda relativa a la sensibilidad al alcance de la mejora tal y como ya ocurría en el análisis univariante.

- Se estima un coeficiente negativo significativo al $1 \%$ para la variable edad. Esto significa que cuanto mayor es la persona, menos está dispuesto a pagar. Conviene señalar que hemos encontrado una correlación significativa al $1 \%$ entre la edad y el nivel de ingresos; esta correlación es positiva hasta los 60 años y negativa a partir de dicha edad, probablemente debido a la jubilación.

- Es interesante lo que ocurre con la variable "recuerda su última factura del agua". Las personas que recuerdan su última factura de agua (variable que se incluyó para detectar la posible referencia monetaria de los encuestados) manifestaron estar dispuestos a pagar mayores cantidades de dinero en relación con aquellos que no la recuerdan (variable positiva significativa al 1\%).

- Finalmente, encontramos que la zona de residencia del encuestado influye en el montante que está dispuesto a pagar por mejorar la calidad del agua en el conjunto de la Cuenca. Es significativo que esto no ocurra en cuanto a la disposición a participar en el mercado contingente, de modo que podemos descartar que los residentes de algunas zonas estén menos dispuestos que los residentes de otras zonas a pagar en principio por dicha mejora, pero sí existe una sensibilidad entre los residentes de distintas zonas en cuanto a la cantidad de dinero que se está dispuesto a pagar, confirmándose la hipótesis tercera. En el modelo estimado observamos como los residentes de las zonas Alto y Valle están dispuestos a pagar significativamente más que los residentes de la zona Campiña (que sirve de línea de referencia en el análisis). Esto puede deberse a que la zona Campiña es la que presenta una peor situación de status quo que el resto de las zonas (ver Cuadro 2), de manera que puede haber cierto "rechazo" (que no es tal rechazo sino disponibilidad a pagar menos cantidad de dinero) a mejorar la calidad de la cuenca en su conjunto (podría especularse: ¿por qué debo pagar para mejorar toda la cuenca si los que estamos verdaderamente mal somos nosotros -habitantes de la zona Campiña-?, las otras zonas no están tan mal, por tanto no necesitan mejorar tanto como nosotros). 


\section{CUADRO 6}

Resultados del modelo de Heckman primera fase: modelo de auto-selección muestral

\begin{tabular}{|c|c|c|c|c|c|}
\hline & Codificación & Coef. & Error típico & $\mathbf{P}>\mathbf{z}$ & I.C. $(95 \%)$ \\
\hline \multicolumn{6}{|c|}{$\begin{array}{l}\text { Regresión Probit de la } 1=\text { participa } \\
\text { variable "Participación" } 0=\text { protesta }\end{array}$} \\
\hline Ingreso medio hogar & $\begin{array}{l}\text { Variable continua (de } \\
0,499 \text { a } 6 \text { miles } € \text { ) }\end{array}$ & 0,038 & 0,012 & 0,002 & $0,014-0,062$ \\
\hline Hijos & $\begin{array}{l}0=\text { no tiene hijos } \\
1=\text { tiene hijos }\end{array}$ & 0,098 & 0,025 & 0,000 & $0,048-0,149$ \\
\hline Nivel educativo & $\begin{array}{l}0=\text { sin educación formal } \\
1=\text { primaria } \\
2=\text { secundaria } \\
3=\text { superior }\end{array}$ & 0,026 & 0,015 & 0,011 & $0,006-0,046$ \\
\hline $\begin{array}{l}\text { El agua es un } \\
\text { problema }\end{array}$ & $\begin{array}{l}0=\text { no en absoluto } \\
1=\text { no es un problema } \\
2=\text { más o menos } \\
3=\text { es un problema } \\
4=\text { es un gran problema }\end{array}$ & 0,066 & 0,015 & 0,000 & $0,036-0,096$ \\
\hline $\begin{array}{l}\text { Importancia de la } \\
\text { calidad del agua }\end{array}$ & $\begin{aligned} 0= & \text { no importante en } \\
& \text { absoluto } \\
1= & \text { no es importante } \\
2= & \text { más o menos } \\
3= & \text { es importante } \\
4= & \text { es muy importante }\end{aligned}$ & 0,081 & 0,013 & 0,000 & $0,055-0,106$ \\
\hline $\begin{array}{l}\text { Grado de conciencia } \\
\text { sobre status quo }\end{array}$ & $\begin{aligned} 0= & \text { no consciente en } \\
& \text { absoluto } \\
1= & \text { no consciente } \\
2= & \text { más o menos } \\
3= & \text { consciente } \\
4= & \text { muy consciente }\end{aligned}$ & 0,029 & 0,012 & 0,013 & $0,006-0,052$ \\
\hline $\begin{array}{l}\text { Credibilidad } \\
\text { escenario status quo }\end{array}$ & $\begin{aligned} 0= & \text { no creíble en } \\
& \text { absoluto } \\
1= & \text { no creíble } \\
2= & \text { más o menos } \\
3= & \text { creíble } \\
4= & \text { muy creíble }\end{aligned}$ & 0,066 & 0,012 & 0,000 & $0,042-0,091$ \\
\hline
\end{tabular}

El signo positivo de Lambda indica que si no se tiene en cuenta el sesgo de autoselección muestral tendría lugar una sobrestimación de la disponibilidad a pagar, ya que los factores que hacen más probable la participación están asociados con mayo- 


\section{CUADRO 7}

Resultados del modelo de Heckman segunda fase: modelo principal

\begin{tabular}{|c|c|c|c|c|c|}
\hline & Codificación & Coeficiente & Error típicio & $\mathbf{P}>\mathbf{z}$ & I.C. $(95 \%)$ \\
\hline $\begin{array}{l}\text { Two-stage least } \\
\text { squares regres- } \\
\text { sions variable } \\
\text { "DAP" }\end{array}$ & Variable continua & & & & \\
\hline $\begin{array}{l}\text { Calidad del } \\
\text { agua }\end{array}$ & $\begin{array}{l}0=\text { calidad buena } \\
1=\text { calidad muy buena }\end{array}$ & 0,024 & 0,081 & 0,762 & (-) $0,135-0,185$ \\
\hline $\begin{array}{l}\text { Ingreso medio } \\
\text { hogar }\end{array}$ & $\begin{array}{l}\text { Variable continua (de } \\
0,499 \text { a } 6 \text { miles } € \text { ) }\end{array}$ & 0,199 & 0,048 & 0,000 & $0,105-0,294$ \\
\hline Hijos & $\begin{array}{l}0=\text { no tiene hijos } \\
1=\text { tiene hijos }\end{array}$ & 0,412 & 0,093 & 0,000 & $0,230-0,594$ \\
\hline Edad & $\begin{array}{l}\text { Variable continua (desde } \\
18 \text { años) }\end{array}$ & $-0,012$ & 0,002 & 0,000 & (-) $0,016-(-) 0,008$ \\
\hline Urbano & $\begin{array}{l}0=\text { residencia zona rural } \\
1=\text { residencia zona urbana }\end{array}$ & $-0,300$ & 0,113 & 0,008 & (-) $0,522-(-) 0,078$ \\
\hline $\begin{array}{l}\text { Residencia en } \\
\text { zona Alto }\end{array}$ & $\begin{array}{l}0=\text { residencia en otra zona } \\
1=\text { residencia en Alto }\end{array}$ & 0,382 & 0,123 & 0,002 & $0,141-0,624$ \\
\hline $\begin{array}{l}\text { Residencia en } \\
\text { zona Valle }\end{array}$ & $\begin{array}{l}0=\text { residencia en otra zona } \\
1=\text { residencia en Valle }\end{array}$ & 0,390 & 0,112 & 0,001 & $0,192-0,625$ \\
\hline $\begin{array}{l}\text { Grado de } \\
\text { conciencia sobre } \\
\text { status quo }\end{array}$ & $\begin{aligned} 0= & \text { no consciente en abso- } \\
& \text { luto } \\
1= & \text { no consciente } \\
2= & \text { más o menos } \\
3= & \text { consciente } \\
4= & \text { muy consciente }\end{aligned}$ & 0,128 & 0,043 & 0,003 & $0,042-0,214$ \\
\hline $\begin{array}{l}\text { Recuerda factura } \\
\text { del agua }\end{array}$ & $\begin{array}{l}0=\text { no recuerda } \\
1=\text { recuerda }\end{array}$ & 0,301 & 0,085 & 0,000 & $0,148-0,483$ \\
\hline $\begin{array}{l}\text { Lambda (razón } \\
\text { Mill inversa) }\end{array}$ & & 0,314 & 0,047 & 0,000 & $0,222-0,407$ \\
\hline $\begin{array}{l}\text { Resumen } \\
\text { modelo: } \\
\mathrm{N}^{22}\end{array}$ & Wald Chi2(10) & $\begin{array}{l}1016 \text { (no cen- } \\
\text { suradas: } 635 \text { ) }\end{array}$ & - $1.366,94$ & Prob > & Chi2 \\
\hline
\end{tabular}

Fuente: Elaboración propia.

res disposiciones a pagar. Esto es, si no se tuviera en cuenta la diferencia entre la disposición a pagar en principio y la cantidad que se está dispuesto a pagar, se incluirían en el modelo de DAP una serie de factores que no necesariamente afectan a la canti-

${ }^{22}$ El número de observaciones es: 1.238 (619 individuos $\times 2$, para el nivel de calidad bueno y nivel de calidad muy bueno) menos los valores "perdidos" (missing values) 
dad de dinero que se pagaría, sino a la disponibilidad a participar en el mercado contingente, sobreestimando la $\mathrm{DAP}^{23}$.

\section{Conclusiones}

El nuevo contexto político para la gestión del agua auspiciado por la Directiva Marco del Agua abre la posibilidad a una gestión sostenible de los recursos hídricos. Bajo esta nueva concepción, las herramientas y principios económicos se ponen al servicio del logro de fines ecológicos como instrumentos informadores del proceso participativo de decisión.

Se supone de manera generalizada que la Directiva generará beneficios ambientales sustanciales. Se han realizado, sin embargo, escasas valoraciones económicas de dichos beneficios, particularmente en lo que concierne a los beneficios de no mercado. Dicha valoración es, no obstante, un requisito necesario en el proceso de implementación de la DMA, ya que ésta misma recoge la posibilidad de derogación de los objetivos ecológicos por motivos de desproporcionalidad de los costes en los que será necesario incurrir para alcanzar dichos objetivos. Es por tanto necesario estimar dichos beneficios para poder informar a los decisores públicos en su evaluación de tal desproporcionalidad.

Tanto la definición de beneficio ambiental como los mecanismos para estimarlos no quedan claramente definidos ni en el texto de la Directiva ni en las interpretaciones posteriores, ni se ha alcanzado todavía en el ámbito europeo a un consenso en este sentido. Esta investigación trata de dar respuesta a esta necesidad surgida específicamente de la implementación de la norma europea. Para ello se ha llevado a cabo un caso práctico aplicado a la Demarcación Hidrográfica del Guadalquivir, basado en la valoración de la disponibilidad a pagar del público (residentes de la Cuenca) por el paso desde el status quo a una situación de calidad del agua mejorada, mediante el planteamiento de un escenario de valoración basado en la definición de la calidad del agua en función de la potencialidad de su uso y el riesgo para las personas y el ambiente y en la división territorial de la Cuenca.

Los resultados de tal aplicación, muestran el interés de los habitantes de la Cuenca del Guadalquivir en la mejora de la calidad del agua y que dicho interés se traduce en una disponibilidad a pagar positiva por dicho cambio ambiental. Concretamente, los valores de DAP obtenidos indican que la población de la Cuenca estaría dispuesta a aumentar de media en torno a un $30 \%$ su factura actual del agua ${ }^{24}$ para obtener una

23 Del mismo modo y en sentido contrario, si Lambda hubiera resultado significativo negativo, la no consideración de la auto-selección muestral llevaría a subestimaciones de la DAP ya que se incluirían en el modelo variables asociadas con una menor disponibilidad a participar, pero no necesariamente a pagar menos cantidad de dinero.

${ }^{24} \mathrm{El}$ informe de recuperación de los costes de los servicios del agua llevado a cabo en una primera aplicación del artículo 5 de la DMA (Confederación Hidrográfica del Guadalquivir, 2007) cifra el pago 
calidad buena o muy buena (en los términos definidos en este estudio). Consideramos que estas son implicaciones interesantes para la política hídrica ya que otra de las probables consecuencias de la DMA será la más que probable revisión al alza de las tarifas de los servicios del agua. En este sentido, es también interesante el hecho de que las personas que recuerdan su factura del agua se muestran más dispuestas a aumentarla. Existe un importante desconocimiento acerca del "precio" real del agua (una parte muy importante de la población no recuerda su factura del agua), que puede estar propiciando una percepción sobredimensionada del mismo.

La agregación de las DAP individuales indica que la mejora de la calidad de la Cuenca en el Guadalquivir generará beneficios anuales en un rango entre los 48 y 59,5 millones de euros ${ }^{25}$, los cuales deberán ser comparados con los costes de las medidas necesarios para alcanzarlos. En este punto conviene sin embargo hacer las siguientes puntualizaciones importantes. Por una parte, esta cifra concierne exclusivamente a los beneficios no de mercado, por lo que deberán añadirse a la misma los beneficios que se generen como consecuencia de la mejora de la calidad del agua y que tengan un reflejo en actividades comerciales (e.g. reducción de los costes de depuración o aumento de la rentabilidad en la agricultura). Además, en esta investigación la calidad ambiental ha sido definida exclusivamente en términos cualitativos y no se han tenido en cuenta los aspectos cuantitativos (caudal, escasez), por lo que estos beneficios calculados pueden considerarse un límite inferior.

En cuanto al aspecto territorial, la DMA requiere el alcance del buen estado ecológico a nivel de Cuenca. En este trabajo hemos establecido la hipótesis de que los beneficios ambientales no estarán homogéneamente distribuidos entre los habitantes de distintos territorios de la Cuenca. Se han aportado pruebas sobre tal distribución territorialmente heterogénea mediante la distinta valoración que los residentes de distintas zonas dentro de la Cuenca hacen de la mejora global de la misma. Dos posibles interpretaciones no excluyentes pueden hacerse en este sentido; por una parte, la diferencia en el status quo de las distintas zonas implica mejoras ambientales de distinta magnitud entre los territorios. Por otra parte, también cabe pensar en la existencia de una cierta heterogeneidad territorial de las preferencias, por ejemplo, una especial valoración de la mejora de la calidad del agua en la zona de residencia del individuo frente a otras zonas dentro de la misma Cuenca. Un análisis específico de esta heterogeneidad territorial de las preferencias (que se podría realizar mediante la definición de zonas como atributos de valoración en un experimento de elección) debería

actual de los usuarios por el saneamiento en unos 40 euros por habitante y año. Los beneficios derivados de la mejora de la calidad del agua calculados en este estudio van de los 31,8 a los 39,42 euros como media por hogar; teniendo en cuenta una media de 2,8 personas por hogar según las estadísticas oficiales (Instituto de Estadística de Andalucía, 2008) estos beneficios alcanzan entre los 11,57 y los 14 euros por persona y año para el nivel bueno y muy bueno.

${ }^{25}$ La agregación de los beneficios se hace teniendo en cuenta las estimaciones medias de DAP calculadas en este estudio que se suman para un total del 4.226.167 habitante de la Cuenca, teniendo en cuenta el porcentaje de respuestas protestas detectado en este estudio. Como se ha mencionado anteriormente, no se descarta que el conjunto de beneficiarios de la mejora de la calidad del agua del Guadalquivir exceda de los límites de la demarcación. 
permitir analizar la interacción de las características del individuo con el lugar específico donde ocurre el cambio ambiental.

Finalmente, en este estudio se demuestra cómo las variables relacionadas con el interés y preocupación de los individuos por el medio ambiente en general y por el agua en particular influyen en la percepción de los beneficios ambientales de la mejora de la calidad del agua. Este hecho, así como el relativo a la percepción sobre la propia factura del agua que hemos mencionado anteriormente, ponen de manifiesto a nuestro juicio la necesidad de que el proceso de implementación de la DMA, sobre todo si va a derivar como es previsible en un reajuste de las tarifas del agua, se haga acompañado de campañas de participación pública, publicidad y concienciación, tanto para legitimar el proceso como para poder estimar de manera más ajustada los beneficios ambientales ante la evaluación de la desproporcionalidad de las medidas.

\section{Bibliografía}

AEAS. (2003). Encuesta de Tarifas 2002. http://hispagua.cedex.es/documentacion/documentos/tarifas_2002.pdf

Amemiya, T. (1984). "Tobit models: A survey". Journal of Econometrics, 24(1-2):3-61.

Arrow, K., Solow, R., Porteny, P.R., Leamer, E.E., Radner, R. y Schuman, H. (1993). "Report of the NOAA Panel on Contingent Valuation". Federal Register, USA.

Baker, B., Metcalfe, P., Butler, S., Gueron, Y., Sheldon, R. y East, J. (2007). "Report on the Benefits of Water Framework Directive Programs of Measure in England and Wales". Department of Environment, Food and Rural Affairs, United Kingdom.

Balistreri, E., McClelland, G., Poe, G. y Schulze, W.D. (2001). "Can hypothetical questions reveal true values? A laboratory comparison of dichotomous choice and open-ended contingent values with Auction Values". Environmental and Resource Economics, 18(3):275-284.

Bateman, I.J., Brouwer, R., Davies, H., Day, B.H., Deflandre, A., Falco, S.D., Georgiou, S., Hadley, D., Hutchins, M., Jones, A.P., Kay, D., Leeks, G., Lewis, M., Lovett, A.A., Neal, C., Posen, P., Rigby, D. y Turner, R.K. (2006). "Analyzing the agricultural costs and nonmarket benefits of implementing the Water Framework Directive". Journal of Agricultural Economics, 57(2):221-237.

Bateman, I.J, Carson, R.T., Day, B., Hanemann, M., Hanley, N., Hett, T., Jones-Lee, M., Loomes, G., Mourato, S., Özdemiroglu, E., Pearce, D.W., Sugden, R. y Swanson, J. (2002). Economic Valuation with Stated Preference Techniques. A Manual. Edward Elgar Publishing, Cheltenham.

Bateman, I.J. y Langford, I. (1997). "Non-user's willingness to pay for a National Park: An application and critique of the contingent valuation method". Regional Studies Cambridge, 31:571-583.

Bateman, I.J., Langford, I.H., Turner, R.K., Willis, K.G. y Garrod, G.D. (1995). "Elicitation and truncation effects in contingent valuation studies". Ecological Economics, 12(2):161179.

Birol, E., Karousakis, K. y Koundouri, P. (2006). "Using economic valuation techniques to inform water resources management: A survey and critical appraisal of available techniques and an application". Science of the Total Environment, 365(1-3):105-122. 
Brander, L., Florax, R. y Vermaat, J. (2006). "The empirics of wetland valuation: A comprehensive summary and a meta-analysis of the literature". Environmental and Resource Economics, 33(2):223-250.

Brouwer, R. (2004). "The Value of Clean Water. Perception and Valuation of the Benefits Associated with the Implementation of the Water Framework Directive in the Netherlands". RIZA rapport 2004-013. RIZA, Lelystad, The Netherlands.

Brouwer, R., Langford, L. H., Bateman, I.J. y Turner, R.K. (1999). “A meta-analysis of wetland contingent valuation studies". Regional Environmental Change, 1(1): 47-57.

Carson, R.T. (1997). "Contingent valuation surveys and tests of insensitivity to scope". En Kopp, R.J., Pommerhene, W. y Schwartz, N. (Eds.): Determining the Value of Non-marketed Goods: Economic, Psychological and Policy Relevant Aspects of Contingent Valuation Methods. Kluwer, Boston: 127-163.

Carson, R.T., Flores, N.E. y Meade, N.F. (2001). "Contingent valuation: Controversies and evidence”. Environmental and Resource Economics, 19(2):173-210.

Carson, R.T., Wright, J., Alberini, A., Carson, N. y Flores, N. (1995). A Bibliography of Contingent Valuation Studies and Papers. Natural Resource Damage Assessment. Inc. La Jolla, California.

Carson, T. y Mitchell, R. (1993). "The value of clean water: the public's willingness to pay for boatable, fishable and swimmable quality water". Water Resources Research, 29(7):24452454.

Comisión Europea. (2003). Common Implementation Strategy for Water Framework Directive (2000/60/EC) Guidance Document no. 8. Public Participation in Relation to the Water Framework Directive. Comisión Europea, Working group 2.9 Public Participation. Luxembourg.

Confederación Hidrográfica del Guadalquivir. (2005). Informe Artículos 5 y 6 de la Directiva Marco del Agua.

http://www.chguadalquivir.es/chg/opencms/dma/menu_izquierda/informe/contenido.html.

Costanza, R., D’Arge, R., Groot, R., Farber, S., Grasso, M., Hannon, B., Limburg, K., Naeem, S., O'Neil, R., Paruelo, J., Raskin, R., Sutton, R. y Van den Belt, M. (1997). "The value of the world's ecosystem services and natural capital". Nature, 387:253-260.

Ferrer, G. y La Roca, F. (2006). "El papel de la economía en el desarrollo e implementación de la Directiva Marco del Agua. ambigüedad conceptual y problemas prácticos”. Mimeo, Universidad de Valencia, Valencia.

Foster, V. y Mourato, S. (2003). "Elicitation format and sensitivity to scope". Environmental and Resource Economics, 24(2):141-160.

Gómez, C.M. (2006). "Los costes y beneficios ambientales en el proceso de decisión de la DMA". Conferencia en la Hydronomic Convention, Barcelona.

Green, C. (2003). Water Economics, Principles and Practice. Wiley, West Sussex.

Gutiérrez, C., Martín-Ortega, J. y Berbel, J. (2008). "Situación y tendencias del uso agrícola del agua en la Cuenca del Guadalquivir. Revista de Estudios Agrosociales y Pesqueros (en prensa).

Halstead, J.M., Lindsay, B.E. y Brown, C.M. (1991). "Use of the Tobit model in contingent valuation: experimental evidence from the Pemigewasset Wilderness Area". Journal of Environmental Management, 33(1):79-89.

Hanley, N., Ryan, M. y Wright, R. (2003). "Estimating the monetary value of health care: Lessons from environmental economics". Health Economics, 12:3-16.

Hanley, N., Wright, R. y Álvarez-Farizo, B. (2006). "Estimating the economic value of improvements in river ecology using choice experiments: an application to the water framework directive". Journal of Environmental Management, 78:183-193. 
Hanley, N. (1996). "Ethical beliefs and behavior in contingent valuation surveys". Journal of Environmental Planning and Management, 39(2):255-272.

Hanley, N. y Wright, J. (1992). "Valuing the environment: recent uk experience and an application to greenbelt land". Environmental Planning and Management, 35(2):145-160.

Heckman, J. (1979). "Sample selection bias as a specification error". Econometrica, 47(1):153-161.

Instituto de Estadística de Andalucía. (2008). Anuario Estadístico de Andalucía. www.juntadeandalucia.es/iea/anuario/

Jorgensen, B.S. y Syme, G.J. (2000). "Protest responses and willingness to pay: Attitude toward paying for stormwater pollution abatement". Ecological Economics, 33(2):251-265.

Jorgensen, B.S., Syme, G.J. Bishop, B .J. y Nancarrow, B.E. (1999). "Protest responses in contingent valuation". Environmental and Resource Economics, 14(1):131-150.

Kallas, Z., Gómez-Limón, J.A. y Barreiro, J. (2007). "Decomposing the value of agricultural multifunctionality: combining contingent valuation and the analytical hierarchy process". Journal of Agricultural Economics, 58(2):218-241.

Kosz, M. (1996). "Valuing riverside wetlands: The case of the Donau-Auen national park". Ecological Economics, 6(2):109-127.

Kramer, R. y Mecer, D. (1997). "Valuing a global environmental good: U.S. residents' willingness to pay to protect tropical rain forests". Land Economics, 73:196-210.

Krutilla, J.V. (1967). "Conservation reconsidered". The American Economic Review, 57(4):777-786.

Loomis, J.B. (2000). "Environmental valuation techniques in water resources decision making". Journal of Water Resources Planning and Management, 126: 339-344.

Maddala, G.S. (1983). Limited Dependent and Qualitative Variables in Econometrics. Cambridge University Press, Cambridge.

Maestu, J., Gómez, C.M., Gutiérrez, C., Martínez, J., Sánchez, M.T., Calvo, I. (Eds.). (2008). Agua y Economía en España: Situación Actual y Perspectivas. Ministerio de Medio Ambiente, Medio Rural y Marino, Madrid.

Martín-Ortega, J. (2008). Beneficios ambientales no de mercado asociados a la implementación de la Directiva Marco del agua: una aproximación territorial a la demarcación hidrográfica del Guadalquivir. Tesis Doctoral, Universidad de Córdoba.

Martín-Ortega, J., Gutiérrez, C. y Berbel, J. (2008). "Caracterización de los usos del agua en la demarcación del Guadalquivir en aplicación de la Directiva Marco de Aguas". Revista de Estudios Regionales, 81: 45-76.

Ministerio de Medio Ambiente, Medio Rural y Marino. (2007). Precios y Costes de los Servicios del Agua en España. Informe Integrado. Madrid.

Mitchell, R. y Carson, N. (1989). "Using surveys to value public goods: The contingent valuation methods". Resources for the Future, Washington DC.

Morrison, M.D., Blamey, R.K. y Bennett, J.W. (2000). "Minimizing payment vehicle bias in contingent valuation studies". Environmental and Resource Economics, 16(4):407-422.

Naciones Unidas. (2003). Informe sobre el Desarrollo de los Recursos Hídricos en el Mundo: Agua Para Todos, Agua Para la Vida. http://www.unesco.org/water/wwap/wwdr/wwdr1/index_es.shtml

Navrud, S. (1992). Pricing the European Environment. Scandinavian University Press/Oxford University Press, Oslo, Oxford.

Pearce, D.W. y Turner, R.K. (1995). Economía de los Recursos Naturales y el Medio Ambiente. Colegio de Economistas de Madrid, Madrid. 
Ready, R., Navrud, S. y Duborg, W. (2001). "How do respondents with uncertain willingness to pay answer contingent valuation questions?". Land Economics, 77(3):315-326.

Ready, R., Whitehead, J. y Blomquist, G. (1995). "Contingent valuation when respondents are ambivalent". Journal of Environmental Economics and Management, 29:181-196.

Riera, P. (1994). Manual de Valoración Contingente. Instituto de Estudios Fiscales, Madrid.

Rowe, R.D., Schulze, W.D. y Breffle, W.S. (1996). “A test for payment card biases”. Journal of Environmental Economics and Management, 31(2):178-185.

Schaafsma, M. y Brouwer, R. (2006). "Overview of Existing Guidelines and Manuals for the Economic Valuation of Environmental Costs and Benefits". EU $6^{\text {th }}$ FP AquaMoney Project: Development and Testing of Practical Guidelines for the Assessment of Environmental and Resource Costs and Benefits in the WFD. www.aquamoney.org.

Seung-Hoon, Y., Seung-Jun, K. y Tai-Yoo, K. (2000). "Dealing with zero response data from contingent valuation surveys: Application of least absolute deviations estimator". Applied Economics Letters, 7(3):181-184.

Shabman, L. y Stephenson, K. (2000). "Environmental valuation and its economic critics". Journal of Water Resources Planning and Management, 126(6):382-388.

Shyamsundar, P. y. Kramer, R.A. (1996). "Tropical forest protection: an empirical analysis of the costs borne by local people”. Journal of Environmental Economics and Management, 31 (2):129-144.

Sigelman, L. y Zeng, L. (1999). "Analyzing censored and sample-selected data with tobit and heckit models". Political Analysis, 8(2):167-182.

Scotland and Northern Ireland Forum for Environmental Research, (2005). The case for valuation studies in the Water Framework Directive. Report WFD55.

Strosser, P. (2006). Assessing Environmental Costs in the Context of the Implementation of the European Union Framework Directive. Conferencia en la Hydronomic Convention, Barcelona.

Tobin, J. (1958). "Estimation of relationships for limited dependent variables". Econometrica, 26(1):24-36.

UNESCO, (2002). 21 st Century Talks: the Future of Water. Paris. http://www.wateryear2003.org

Wesley, A.M., Joel, H., Viscusi, W.K. y Jason, B. (2000). "An iterative choice approach to valuing clean lakes, rivers, and streams". Journal of Risk and Uncertainty, 21(1):7-43.

Whitehead, J., Groothuis, P.A. y Blomquist, G.C. (1993). "Testing for non-response and sample selection bias in contingent valuation: Analysis of a combination phone/mail survey". Economics Letters, 41(2):215-220.

Wittmer, H., Rauschamyer, F. y Klauer, B. (2006). "How to select instruments for the resolution of environmental conflicts?". Land Use Policy, 23:1-9.

World Wide Fund for Nature and European Bureau. (2006). Survey of the economic elements of the Article 5 report of the EU Water Framework Directive. Brussels. 
4-Martin Ortega 4/2/09 20:14 Página 90 